\title{
POINT DEFECTS IN NON-METALLIC SOLIDS
}

$\mathrm{D}$ IAMONDS, graphite, oxides and phosphides, zeolites and alumino-silicates all came up for discussion at a conference at Leamington Spa organized by the British Ceramic Society through its Basic Science Committee during October 14-16, 1963. The wisdom of making the programme so wide as regards materials examined, and so well defined in limiting the discussions to the properties of point defects, was exemplified by the liveliness of the discussions and the encouraging overlap of the technical terms now current in the field. Despite the wide selection of materials the majority of the audience was interested for the majority of the time. From the point of view of a physicist, one of the most encourag. ing features was the growth of the recognition that the basic properties of any pure material need to be isolated from those deriving from the defects it contains. This recognition has spurred on the production of purer materials for investigation. Of course, the example of semi-conductors and the immediate pressure of laser and maser requirements has given a real edge to these considerations, and the preparation of large, highly pure crystals of many halides and a fow oxides is now possible.

There are two kinds of purity to be discussed for these ionic substances; chemical contamination by extraneous material must be avoided, but also, perhaps less obviously, the proper proportions of the main constituents to produce a stoichiometric crystal must be maintained. It was with two papers on this topic that the conference opened. It was shown that careful density and lattice parameter work can give information on the numbers of vacant lattice sites, and that extra diffraction lines may betray complex ordering behaviour of the defects. The advantages of neutron diffraction over X-rays were brought out clearly by investigations of oxygen defects in compounds between $\mathrm{UO}_{2}$ and $\mathrm{U}_{4} \mathrm{O}_{9}$. Another paper in this section showed how the electron microscope can identify small clusters of defects as interstitial atom groups rather than vacancy aggregates.

The difficulties of positive identification of individual defect centres in II-VI compounds were outlined, but the conference quickly settled down on the second day to detailed discussion of recent experimental information on alkali halides, diamond, magnesia and rutile. Most of these papers sought to set up models to account for the electron spin resonance or optical properties. In diamond, magnesia and calcium fluoride, many of the optioal bands have now been shown to have sharp, zero-phonon, lines at the high-energy edge of the band. It is often of good intensity and, in one case at least, was split by application of stress to the diamond. There seems no doubt that such lines will repay more detailed investigation than has been possible on the broad bands seen hitherto.

Coloration in rutile under irradiation has been investigated at Brookhaven Laboratories, but virtually no specific effects in the optical spectrum can be identified. A general small rise in absorption is all that has been seen in room temperature irradiation experiments on crystals of carefully controlled stoichiometry. Photoconduction peaks were, however, produced by neutron irradiation.

Impurity coloration due to cupric ion in a number of solvent crystals- $\mathrm{ZnO}, \mathrm{MgO}$ and $\mathrm{ThO}_{2}$ - was interpreted as demonstrating marked differences in the ion's electric levels in crystal fields of different symmetries. The effects of tetragonal Jahn-Teller distortions from cubic symmetry were identified and values of the crystal field splitting parameter tabulated. The explanation of the susceptibility of the cupric oxide crystal itself seems to demand some significant antiferromagnetic coupling of pairs of spins even in the paramagnetic state.

The next section of the meeting was concerned with atomic mobility in solids. It was interesting to have some discussion at last of the role of anion vacancies and interstitials in simple ionic crystals, and some experimental evidence that they could not be permanently ignored was presented. The analysis of diffusion mechanism has always been fruitful in identifying the most mobile of the possible atomic defects, and it is most impressive now to hear of the delicacy and accuracy of the experiments which allow the relative rates of motion of different isotopes to be followed. The measurements on ${ }^{22} \mathrm{Na}$ and ${ }^{24} \mathrm{Na}$ in sodium chloride have shown again that the most exacting test is still satisfied by the idea that anion vacancy diffusion is dominant. That the fluctuation in the kinetic energy to carry the system to the 'activated complex' state resides mainly on the jumping atom is established by the closeness with which the basic rates are proportional to the square root of the mass of the jumping atom. The discussion brought to light some interesting measurements from the Imperial College of Science and Technology on the charges absorbed on dislocations, whch were interpreted as a measurement of the different partial supersaturation of positive and negative ion vacancies in sodium chloride, and hence of their energies of formation. Despite some reservations as to the rigour of the argument, this seemed a promising new departure. Another short contribution from the same group showed how the temperature range, where diffusion rates are intrinsic, can be extended in the alkali halides by adding oxide or sulphate ions to depress the number of non-thermal free positive-ion vacancies. The observed absence of a reduction in diffusion coefficient below the intrinsic line is hard to understand. This section closed with an address dealing with the significance of the non-equilibrium thermodynamic formulation of atomic mobility, which showed that, despite (or because of ?) its generality, it was easy to fall into error by failing to define carefully all the variables and their interdependences. For example, vacancy flux must be introduced with care since it can be deduced from atomic fluxes for some boundary conditions and not for others. It was concluded that it was worth while to develop some familiarity with the formation by translating the results of some atomic models into the language of irreversible thermodynamics, before trying to use the technique in unfamiliar situations.

Prof. Barrer gave a succinct guide to the features controlling ionic diffusion in various zeolite-type structures. In some of these, wide continuous channels exist and diffusion barriers are essentially electrostatic in nature, but in others, narrow points separate atomic cages, and repulsive forces come into play. The connexions between surface chemistry, surface diffusion, and bulk diffusion became obvious, and the practical importance of interdisciplinary conferenees of this kind was again emphasized in the minds of those present.

The intricate question of how ionic is a salt was raised by a paper on the E.M.F. of the diffusion cell. It was shown that the transport of charge during diffusion of, say, aluminium atoms through alumina is not necessarily precisely that corresponding to the motion of $\mathrm{Al}^{+++}$ions. The actual value depends on tho precise mechanisms of compensation of the charges of the defects responsible for diffusion. 
The final section related to the mechanical properties of ionic solids, as affected by point dofect. The introductory lecture by Prof. Pratt showed cloarly that two main categories of effects could be considered; the direct effects of defects or their aggregates, and the indirect effect of tho interaction of the defects with pre-existing dislocation or dislocations formed by mechanical deformation. It appears that in some circumstances a main effect may be the indirect pinning of dislocations by their electrostatic or stress interaction with ro-oriontable dipole-type defects. A short paper on sodium chloride with controlled concentration of divalent cations supported this view. The nature of the relaxation of dipoles formed by trapped defects was discussed in group theoretical terms in the next paper, and it was shown that except for very short times a single relaxation time appropriate to noarest neighbour orientations of the defect is reasonably accurate.

The effects of irradiation on the plastic properties of sodium chloride showed a rise in yield stress, a tendency to smaller slip-step heights and to simultaneous slip on equivalent planes with suppression of kinking. None of the changes was related to $F$-band colour, but $V$ band and interstitial density were not checked. The effects observed in the Youngs modulus of polycrystalline graphite after low-temperature electron irradiation appeared to be due to the pinning of dislocations, but no detailed mechanism could be proposed.

Electron microscope examinations have been made on a number of deformed and defective crystals. The development of oxygen deficiency in titanium dioxide helps the annealing out of stacking faults by a mechanism which may well involve the dissolution of titanium interstitial atoms, after condensation of oxygen vacancies has eliminated planes of oxygen. The titanium interstitials aro in fact identified by colour studies. Other dislocation roactions have also been observed. A separate group reported an increase in optical absorption associated with an increase in titanium interstitial content after hightemperature plastic deformation of an already oxygendeficient titanium dioxide crystal, and also attributed this to either stacking fault elimination or to dislocation climb. In view of the lack of structure in the optical absorption investigations, several comments were made to the effect that an increase in titanium interstitial content could not yet be accepted as proved. Electron microscope examinations were also reported on plastically deformed TiO, and annealing investigations pointed to an activation energy of some $5 \cdot 5 \mathrm{eV}$ for atomic mobility.

To sum up, this was a good conference to be at. It brought together groups of people who meet too seldom, and showed again the unity of the physical sciences. It was the right size-about 120 people-and the programme was not so packed that it was impossible to find a moment to talk to most of those one meant to. The wisdom of holding the conference during university term, when many people found it hard to be away for three days, and when it was not possible to use the lecture theatres of a university, may be queried. A proper lecture room is really quite important, though not nearly so important as the programme, content and participants. I am sure the success of this meeting will encourage the British Ceramic Society to hold more meetings of similar kind to bring these neglected substances under regular scientific review.
W. M. LOMER

\section{MECHANISMS OF ADSORPTION FROM SOLUTION}

$\mathrm{A}^{\mathrm{s}}$ $\mathrm{N}$ informal discussion of the Faraday Society under the above title was held in the Department of Mechanical Engineering, Imperial College of Science and Technology, during September 19-20, by kind permission of Prof. O. A. Saunders. The meoting was attended by 144 people, 81 of whom came from industrial organizations and 63 from academic institutions. There were 16 overseas visitors.

Sir Eric Rideal opened the meeting and, as the first item, Dr. J. J. Kipling (University of Hull) gave a review of the present position in this subject. After outlining the main historical periods, and the progress which was made in each, Dr. Kipling discussed at some length the problems encountered when, instead of an adsorbing vapour, we have to deal with a two (or more) component liquid system. The basic equation relating changes in the surface and bulk mole fractions of the components was given, and its relation to the Gibbs surface excess and the individual adsorption isotherms for the components was described. The use of adsorption from solution for measurement of surface area was examined critically, and, in particular, the difficulty of selecting an appropriate cross-sectional area for the adsorbate molecule was stressed. The difficulty of satisfactorily taking into account localized site adsorption was also discussed. Finally, Dr. Kipling mentioned the question of chemisorption of molecules from solution and foresaw the problem well known in vapour adsorption of distinguishing between chemically and physically adsorbed molecules.

The first paper was given by Prof. D. H. Everett (University of Bristol) and was entitled "Thermodynamic Models of Adsorption from Solution". The adsorption of the components of a binary liquid mixture on to a uniform surface was described in statistical mechanical terms and a new definition of the activity coefficients of the components in the adsorbed layers was proposed.
Prof. Everett showed that the "isotherm of concentration change" may be markedly dependent on the nature of the solution concerned, for example, whether it is a regular solution, or contains molecules of different size. The use of adsorption from solution for the determination of specific surface areas was mentioned and it was suggested that useful results could be obtained from measurements of adsorption from mixtures of molecules of approximately the same size (for example, cyclohexane + benzene).

This was followed by a paper on the "Thickness and Structure of Layers of High Polymers adsorbed from Solutions to Solid Surfaces", by F. Rowland, R. Bulas and Prof. F. R. Eirich (Polytochnic Institute, Brooklyn, New York). The paper was given by Prof. Eirich, who explained the difficulties and uncertainties of investigating the nature of polymer adsorption and then presented results obtained principally by observing the change in flow of liquids through sintered glass disks of known pore size distributions and surface areas. From an analysis of the hydrodynamics of the system, the change in effective radius of the capillaries resulting from polymer adsorption was estimated. Several polymers in different non-aqueous solvents have been examined and it was shown that the film thicknesses were of the same order of magnitude as the diameters of the free coils in solution. A detailed discussion was given of the nature of the adsorption isotherms, of their reversibility and of the influence of good and bad solvents.

A contribution to the investigation of the adsorption of ionized polymers was made by J. B. Craig and Dr. P. Meares (University of Aberdeen) under the title "The Adsorption of Polyacrylates at a Charged Mercury-Salt Solution Interface". Experimental data had been obtained from electrocapillarity investigations, and from derived thermodynamic relations, the surface excess concentrations of polyanions, counter-ions and co-ions 